\title{
Direct multi-objective optimization of parametric geometrical models stored in PLM systems to improve functional product design
}

\author{
J.-B. Bluntzer ${ }^{1}$, S. Gomes ${ }^{1}$, D.H. Bassir ${ }^{2, a}$, A. Varret ${ }^{1}$ and J.C. Sagot ${ }^{1}$ \\ 1 Laboratoire SeT, Equipe ERCOS, University of Technology of Belfort-Montbéliard, 90010 Belfort Cedex, France \\ 2 Institut FEMTO-ST, UMR/CNRS 6174, 24, rue de l'Épitaphe, 25000 Besançon, France
}

Received 9 January 2008, accepted 28 January 2008

\begin{abstract}
This paper presents a functional analysis and design method, including an optimization loop, which has been integrated into our self-developed, web-based Product Lifecycle Management (PLM) platform. Our design methodology, which includes advanced and parametric CAD modelling and direct multiobjective optimization is described here. Functional design and knowledge-based engineering features, such as functional parameters, expert rule definitions and design experience feedback are all developed in the product design process in order to reduce costs, lead time and also to improve product quality and value. To confirm our research hypotheses, an experimental case-study is chosen: the ground-link system of a racing car design and manufacturing project, including conceptual, embodiment, detailed design and manufacturing phases.
\end{abstract}

\section{Introduction}

Concurrent engineering methods and tools are increasingly used within industrial organizations in order to reduce costs, lead time and also to improve product quality and value. The design activity involves many contributors and experts throughout the product lifecycle, which starts with product task identification / functional definition / product modelling / manufacturing and ends with its destruction or recycling. These contributors and experts must collaborate using software, such as PLM (Product Lifecycle Management) systems, which can handle concurrent engineering, in order to help design team members to manage information and knowledge in their project tasks.

Our research activity is to develop a design methodology based on well-known design approach such as "Total design" [1], "Systematic design" [2] or the Ullman mechanical design process [3], but applying direct multiobjective optimization, linked to functional design and knowledge based engineering [4] features, such as expert rule definition or design experience feedback, in order to reduce costs, lead time and also improve product quality and value.

In our global market context, where management, designers, subcontractors and customers are geographically distant from each other, industrial companies have to innovate, often by defining complex products [5] whose design spans various engineering problems and disciplines.

\footnotetext{
a Corresponding author: hbassir@univ-fcomte.fr
}

At the same time, companies have grown in complexity but have also reduced their competencies to be ultimately specialized in few disciplines and thus have to work more extensively with subcontractors. Besides the traditional financial considerations, more recent industrial requirements, such as robustness and performance of the design, but also marketing criteria have come into play and are fast becoming key characteristics of the design. In order to stay competitive, these too must be optimized.

Our design and optimization methodology helps the designer to progress parametric CAD models of an optimized product [6] through functional requirements, design rules and design objectives that can be, respectively, verified and reached using optimization loops. Nowadays, actual real-world engineering design problems involve simultaneous optimization of several objectives and compliance with limiting factors that have been determined by the design team. Engineering design of complex systems is a decision making process that must select from among a set of options leading to an irrevocable allocation of resources. It is inherently a multi-objective process. As products become increasingly complex, their design process, which is geographically distributed [7], is usually characterized by numbers of design variables, parameters, requirements, constraints and objective functions. Taking into consideration the product complexity and the concurrent engineering design context, our methodology is based on a collaborative design process [8] and a multiobjectives optimization loop, using a Product Lifecycle Management (PLM) environment [9]. 


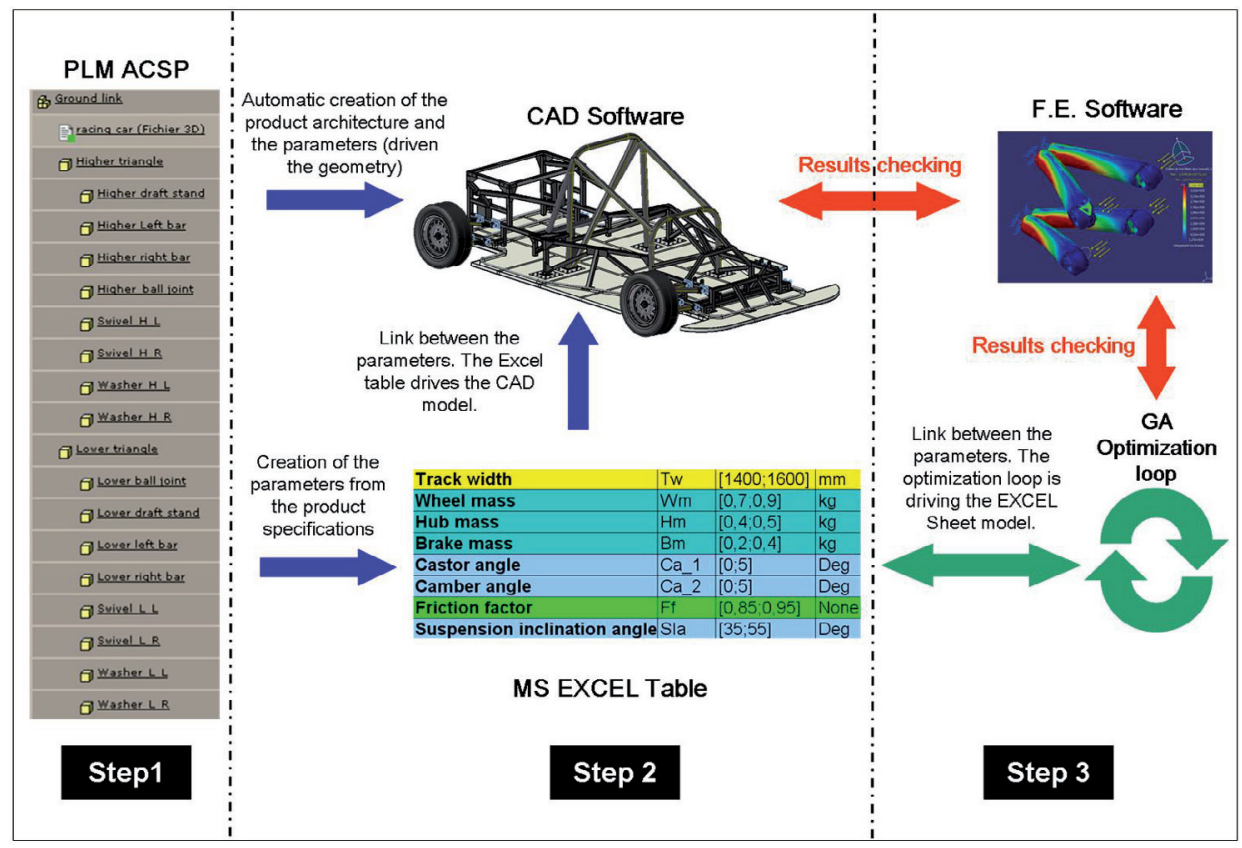

Fig. 1. Global methodology model for collaborative, functional and knowledge-based engineering using a PLM environment and integrating an optimization loop.

After this first presentation of the state of the art and of industrial requirements, we will present our functional and technical analysis and design approach, including an optimization loop, built into our self-developed web-based [10] PLM platform, called ACSP (in French: Atelier Coopératif de Suivi de Projets) [11]. Then, the main principles of multi-objective optimization using genetic algorithms are explained in the third section.

To confirm our research hypotheses, an experimental case study is chosen: the ground-link system of a racing car design and manufacturing project, including conceptual, embodiment and detailed design phases as well as manufacturing phases.

\section{Proposed methodology and tools}

In order to achieve our goals, our methodology includes three different steps as shown in Figure 2.

The first step is carried out by the designer. He has to input the customer specifications into the ACSP PLM system, in order to fill the functional parameter forms. At the same time, he has to establish the product architecture (also called product tree) [12]. In fact, he has to determine three different levels in order to characterize the product:

- Level 1: the global product, which is the assembly of all sub-products.

- Level 2: the sub-products, which are assemblies of parts which are kinematically fixed (no degrees of freedom).

- Level 3: the parts, which are the elementary components of the product.

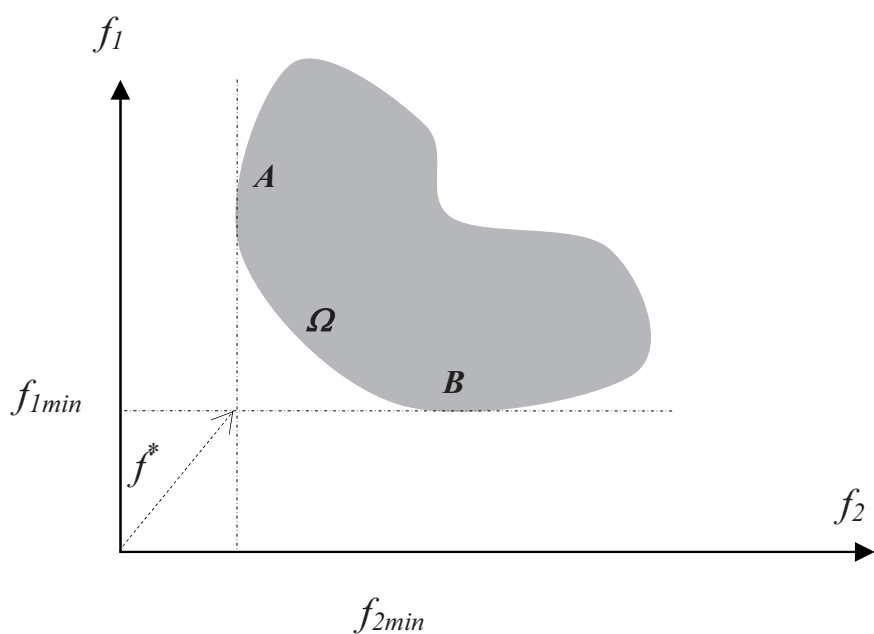

Fig. 2. Pareto curve $\left(\operatorname{Min}\left(f_{1}\right.\right.$ and $\left.\left.f_{2}\right)\right)$.

In the same step, the designer has to register all the design rules, the design terms, etc. in a shared knowledge database.

The second step which can be considered as the core of our methodology, is to carry out a functional/structural parameter (extracted from the first step) propagation taking into consideration the impact of each function (and also the corresponding value criteria, so called functional parameters), in each part of the product. Each parameter input into our CAD model is driven by an MS Excel file, also automatically generated from the PLM system. The main advantage of this kind of method is that we can modify the MS Excel files on other software in order to directly impact the CAD model. 
In order to carry out the parameter propagation in the CAD model product tree, functional parameters of the product (global parameters) are copied into sub-products as local parameters. They are then linked to the previous global parameters through equality equations, in order to:

- generate a constraint/limitation/specification propagation from the top (global product) to the bottom (parts) of the CAD model product tree;

- at least, allow designers to carry out CAD modelling activities on a sample of the product Digital MockUp, after a check-out operation of this product sample (sub-product) using the PLM system. This approach preserves the impact of updated parameters on updated geometry and allows the other designers to work simultaneously on the other sub-products;

- interface our ACSP PLM system with commercial and parametric CAD software such as PTC's Pro/ENGINEER or Dassault Systems' CATIA v5. In this context, two requirements can be considered: data exchange between the PLM environment and the CAD tool, and also visualization of $3 \mathrm{D}$ models, using respectively, SQL and XML technology and VRML, 3DXML, X3D, U3D, DWF, open JT, etc. new CAD file formats, for the latter.

Finally, one last step can be included, as it is to optimize the parameters already defined in the MS Excel files. These parameters are directly sent into the FEM checking software, at each loop of the data computing process, in order to confirm the optimized results. The following figure describes graphically the previously described methodology.

In the next two sections, we will describe the optimization concepts using genetic algorithms and also how we can integrate this methodology in a real design case study that considers not only one but several objective functions at the same time.

\section{Optimization concepts using genetic algorithms}

In this paragraph, we will describe briefly the functioning of the genetic algorithms in the framework of multiobjective or multicriteria optimization.

\subsection{Genetic algorithms}

Genetic algorithm (GAs), is derived, as its name suggests, from natural selection. GAs provide an alternative to traditional optimization techniques for locating the optimal solutions in a complex search landscape. The first to introduce this paradigm is Holland in 1975 [13]. Since that time, many applications and publications had been described and especially after the last works of Goldberg in 1989 [14] which gave GAs this characteristic of providing global and efficient methods of overcoming complex optimization problems $[14,15]$.
The principle of GAs is to simulate the evolution of one population of individuals to which different production operators (selection, crossover and mutation) are applied. As the GAs start searching from different initial solutions, this gives them a global view of the problem. This global perspective prevents them from being trapped locally and allows them to explore the whole search landscape. Such algorithms identify the problem only through the value of the cost function and the values of the constraints. The behaviour of this algorithm is similar to a black box with several entries and one exit [20].

The functioning of GAs can be divided into three main parts $[16,17]$ : the coding of the parameters, the genetic operators and the choice of the objective functions. The general principles can be described as follows:

1. Assume an initial population of size $N$ (containing $N$ individuals or equivalent solutions).

2. Calculate the fitness of each individual in the population. The fitness is a given function related to the objective function.

3. Select randomly individuals from the current selection based on their fitness and form a new selection of size $N$. This is often called the reproduction operator.

4. Recombine the selected new population by identifying two individuals to represent the two parents, then choosing a crossing point and finally exchanging the values to the right of the crossing point in order to create two new sons. The crossing point is a location in the string representing the chromosome associated to each individual. Such step is represented by a crossover operator. It is carried out using a given probability independent of the choice of individuals.

5. The population obtained in the previous step is mutated with a given probability. Mutation is based on the string representation of the individuals. For instance, if the string representing the individual is coded as a sequence of 0 and 1 , mutation is simply a switch of binary values to their opposite ( 0 to 1 and 1 to 0 ). Mutation introduces new individuals that are not in the initial set, which was defined in the first step.

The first problem to be faced during the use of the GAs is the representation of the individuals (coding of the parameters). It is the manner in which each variable of the optimization problem is coded. The coding can be binary coding or real coding $[16,17]$. The binary coding is the common representation that is most often used in GAs. For continuum variables, many authors [18-20] prefer using real coding for its simplicity and efficiency of use in real problems. Real coding avoids the difficulties of achieving arbitrary precision in decision variables and the Hamming Cliff problem associated with binary string representation of real numbers. Whereas, in the case of discrete variables, the binary representation is more or less the unique solution to overcome certain problems. In this paper, real and binary coding were chosen to be implemented in our program.

A wise definition of the objective function is a very important task in the evolution process, because, GAs 
search the landscape for a solution often using only the discrete values of the objective function. If this function is not well defined, the GAs can not guarantee the location of the global solution.

Finally, the common operators used in GAs are selection, crossover and mutation. These operators are widely described in literature. However, in our program GAPS (Genetic Algorithm with Parallel Selection) [20], we have include the sharing concept that is inspired by nature. In a domain, two species can coexist and share the same resources. This idea [23] is introduced artificially in GAs through a sharing function. This function is to expel some individuals from a research-field that is reducing (example of the niches or zones of local minima) while the density of the population is increasing. The sharing function compares the relative distance between two individuals. Even if this function increases the calculation time of the GAs, it is very suitable in many real cases with many local optima because it improves the exploration capabilities of the GAs.

In the case of scalar optimization or mono-objective optimization, the selection operator is easy to implement. However, in the case of multi-objective optimization, the selection operator is based on a different concept $[19,21]$. In the following, we will make a brief introduction to multi-objective optimization and its characteristics, such as how to choose between the direct or post-priory approach and how to handle the limitations.

\subsection{Multi-criteria/multi-objective optimization}

A multi-criteria or multi-objective optimization problem is stated as follows: Find the vector of design variables $\mathbf{x}=$ $\left[x_{1}, x_{2}, x_{3}, ., x_{n}\right]^{T}$ which minimizes the vector of objective functions $F(x)$.

$$
\operatorname{Min} \mathbf{F}(\mathbf{x})=\operatorname{Min}\left[f_{1}(x), f_{2}(x) \ldots . . f_{k}(x)\right] .
$$

Subject to linear or non-linear constraints: $\boldsymbol{g}_{j}(\boldsymbol{x}) \geqslant 0$ $j=1,2, \ldots, m$

The feasible domain (Fig. 3) defined by the constraints will be denoted by $\Omega . f_{i}: \Omega \rightarrow R, i=1,2, . ., k$ are called criteria or objective functions and they represent the design objectives by which the performance is measured. A vector $\mathbf{x}^{*} \in \Omega$ is called Pareto-optimal solution, if there is no vector $\mathbf{x} \in \Omega$ which would decrease some criteria without causing a simultaneous increase in at least one criteria function. Usually several Pareto-optimal solutions exist.

The optimal solution from the Pareto domain can be reached by two main strategies: either by a direct approach or by a Posteriori approach (Fig. 4).

The Direct method is based on the transformation of the initial problem into a single optimization problem. This transformation can be made by using, for example, the weighting method. This reduces a vector-optimization problem to a scalar optimization problem, where for instance the scalar objective function $f(x)$ can be defined as the weighted sum of the individual objective functions. The Pareto-optimal solutions obtained by this method depend on the choice of the weighting factors $\alpha_{i}$ that will
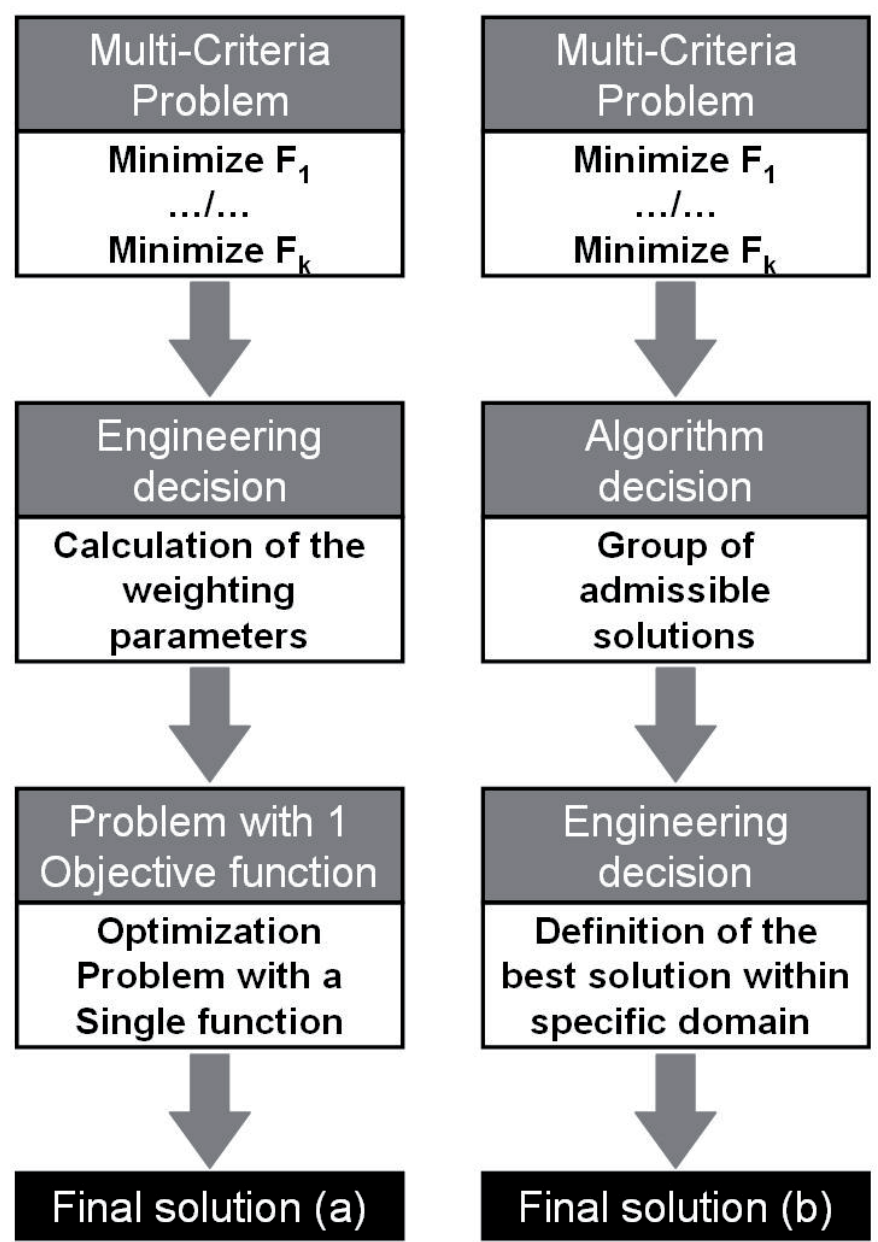

Fig. 3. Direct (a) and Posteriori (b) methods for multiobjective optimization.

generate a unique Pareto optimal solution of the original problem. This approach requires the definition of appropriate weighting factors that will guide the convergence of the optimal design process $[22,23]$. However, this definition depends on the decision-maker and the shape of the Pareto domain (continuity, convexity and the number of limitations to handle). If the weighting factors are not well chosen, this approach can converge into local zone of the Pareto domain, especially when the optimal solution is very sensitive to the weighting factors. This conclusion was described and demonstrated for some academic examples by various authors such as Das [24]. Other researchers have defined good strategies to calculate these weighting factors using sensitivity evaluation of the objective functions which is impossible in the case of the non differential functions. Even when the final solution is reached, there is no guarantee that it is the best one for the decision-maker, as he needs to run the process many times before making his decision.

As concerns the Posteriori method, it starts with a group of initial solutions that we spread uniformly to have a global idea about the Pareto front and to make the final decision easier to take for the decision-maker. In the case 


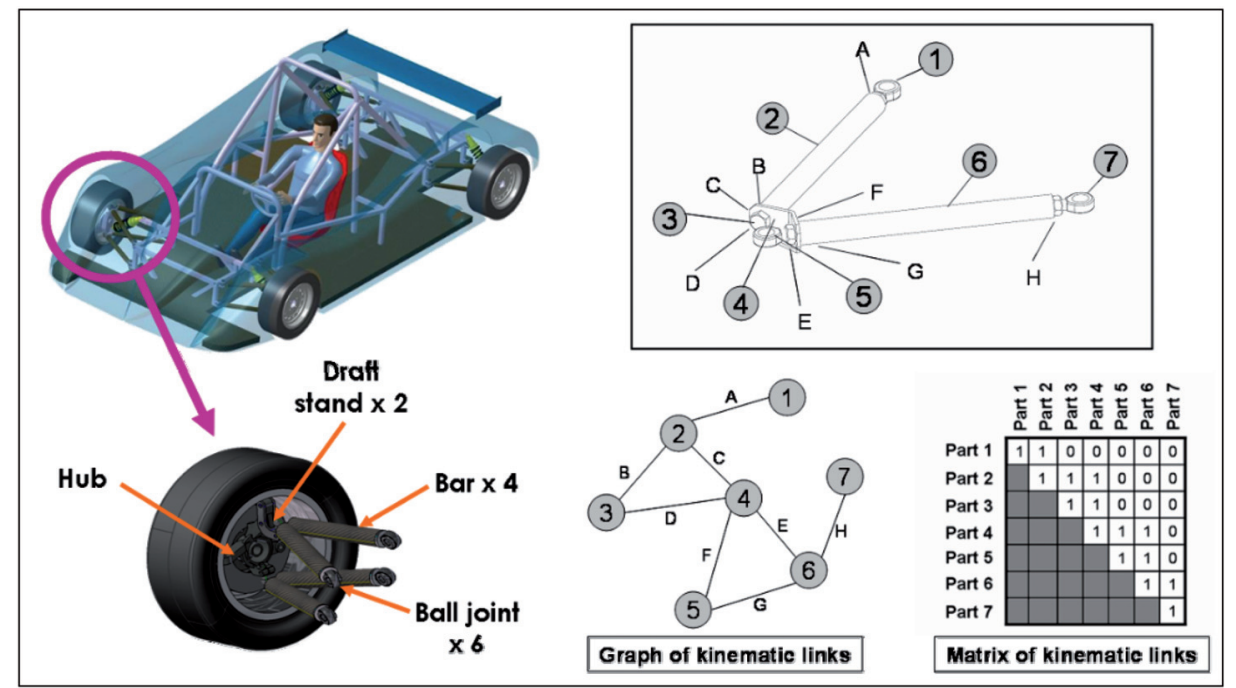

Fig. 4. Ground-link suspension system defined kinematically.

of a composite material, the difficulties in using a simple GA program are, firstly, that the comparison between two solutions can not be achieved easily in the selection process. Secondly, the constraints handling became difficult to represent as an inequality or equality equation. To overcome these difficulties, many researchers like Deb for instance, proposed approaches based on his previous investigations on the genetic algorithms [21]. He introduced the idea of no-dominated sorting to speed the convergence of his algorithm NSGA, to increase its performance and to obtain his actual algorithm NSGA-II. To choose between two solutions $i$ and $j$ in order to continue the iterative process of the GA, a non-dominate sorting approach is used in the selection process as follows.

- Between two admissible solutions $i$ and $j$, we chose the one with the best objective function.

- If solution $i$ is admissible and $j$ non-admissible so we chose the solution $i$.

- If the solutions $i$ and $j$ are non-admissible, we chose the one with minimum violation of the limitations.

To handle the constraints in the GA program, we define the following composite fitness function for any solution $x$ such as:

$$
\begin{aligned}
& F(x)=f(x) \text { if } x \text { is feasible, } \\
& \quad \text { otherwise } F(x)=f_{\max }+C V(x) .
\end{aligned}
$$

$f_{\text {max }}$ is the objective function value of the worst feasible solution in the population and $C V(x)$ is the overall normalized constraint violation of the solution $x$. Thus, there is no need to have any penalty parameter for handling the constraints as usually used in the common approaches. Constraints are normalized to avoid the scaling problems and are equal to one in the case of feasible solution.

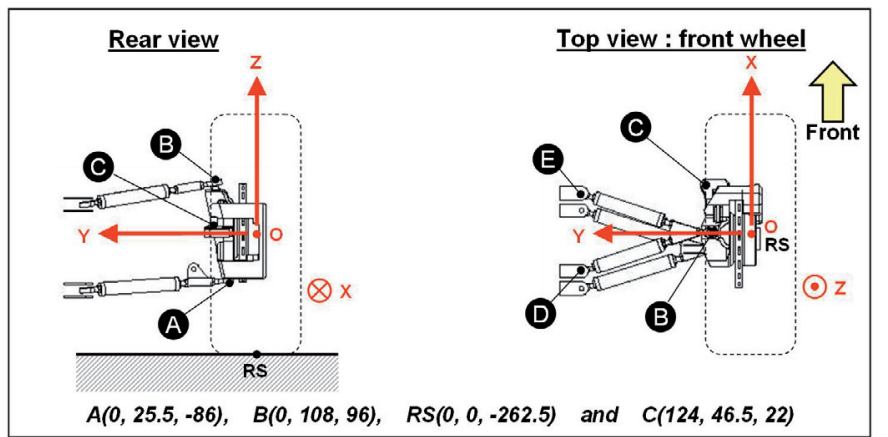

Fig. 5. Geometries of the wheel, hub and the brake.

\section{Experimental design study}

To demonstrate the efficiency of our approach, an experimental design case is chosen. Every year, our mechanical engineering and design department ERCOS has to develop and prototype an entire new racing vehicle. To simplify the demonstration, we choose to limit the experimental case study to a sub-product of the racing car: the ground-link suspension system (Fig. 4). This sub-product of the racing car includes many mechanical parts linking the wheel to the chassis. The design and optimization process will focus on the suspension triangles (wishbones) of a ground-link system. The main steps of our previously presented methodology are applied in this experimental case.

During the optimization process, the technical characteristics of external systems in interaction with the ground link suspension (chassis, hubs, brakes and wheels) have been considered as requirements $(\mathrm{R})$ as follows.

The geometries of the wheel, hub and the brake are given below in Figure 5, with the associated coordinates.

The track width of the vehicle is fixed at $1600 \mathrm{~mm}$, (Castor, camber angles, toe-out are equal to $0^{\circ}$ (simplified configuration of the sets), the mass $m$ of the wheel, hub and the brake is set at $15 \mathrm{~kg}$, the vehicle suspended 
Table 1. Table of the material variation.

\begin{tabular}{|c|c|c|c|c|c|c|c|}
\hline \multirow[t]{6}{*}{ Material } & & cost & & & & & \\
\hline & item & material & $(€ / \mathrm{T})$ & $\rho(\mathrm{kg} / \mathrm{m} 3)$ & $E$ (Gpa) & $R e(\mathrm{Mpa})$ & $n$ \\
\hline & 1 & steel & 700 & 7850 & 210 & 235 & 4 \\
\hline & 2 & aluminium & 4000 & 2900 & 75 & 180 & 4 \\
\hline & 3 & stainless & 4500 & 8700 & 203 & 185 & 4 \\
\hline & 4 & carbon & 5000 & 1530 & 50 & 555 & 1.5 \\
\hline
\end{tabular}

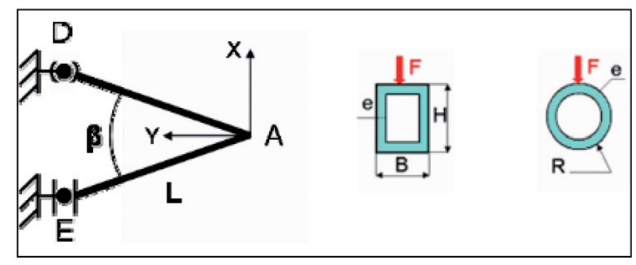

Fig. 6. Technical parameters scheme.

mass $M$ is equal to $460 \mathrm{~kg}$, the front/rear allocation of the suspended mass $\left(M_{v}\right.$ for the front and $M_{r}$ for the rear are respectively equal to $160 \mathrm{~kg}$ and $300 \mathrm{~kg}$ ), the vehicle center of gravity height above the ground $h$ is fixed at $0.35 \mathrm{~m}$, the vehicle wheel base is equal to $2.495 \mathrm{~m}$, the suspension inclination angle in relation to the ground $\alpha_{a m}$ is fixed at $45^{\circ}$ and finally, the friction factor of the tyre on dry road is equal to 0.85 . We should note also that the lower triangles are included in the horizontal plane. For each triangle, the bisectrix of the angle formed by the 2 tie-rods and the vehicle axis is perpendicular.

As concerns the requirements related to the mechanical characteristics of the ground link suspension, they are considered as a technical parameters (T) (Fig. 6). Based on these requirements, we obtain mixed variables for the optimization process (continuous and discrete variables).

The continuous variables are represented by the angle between the tie rods $\beta \in\left[20^{\circ} ; 120^{\circ}\right]$, the external diameter (or side) value $H \in[10 \mathrm{~mm} ; 40 \mathrm{~mm}]$, the thickness $\mathrm{e} \in[1 \mathrm{~mm} ; 3 \mathrm{~mm}]$ and the tie-rod length $L=300 \mathrm{~mm}$. The discrete variables are represented by the section type square shape $(B=H$ or round shape: $H=2 R)$ and the sets of material parameters (Tab. 1).

For the multi-objective optimization problem, we consider the three functions to be minimized that are described below in equations (3) to (5):

$$
\begin{aligned}
& \text { Tie-rod mass } \quad m=\rho S L \\
& \text { Global stresses } \quad \alpha_{\max }=\left|\sigma_{t c}\right|+\left|\sigma_{f}\right| \\
& \text { Global strains } \quad \Delta f=\sqrt{f^{2}+d l^{2}} .
\end{aligned}
$$

The optimization problem had been processed in two steps. First, we applied traditional mechanical analysis of undeformable solids, which enabled the maximum deceleration acceptable by the vehicle and loads generated at the various link points of the suspension triangles to be defined. Then, we defined a standard material strength calculation in order to identify the stresses and deformations corresponding to these loads.

The limitation or constraint $G_{j}$ is represented by the maximum stress allowed in the normal section that should

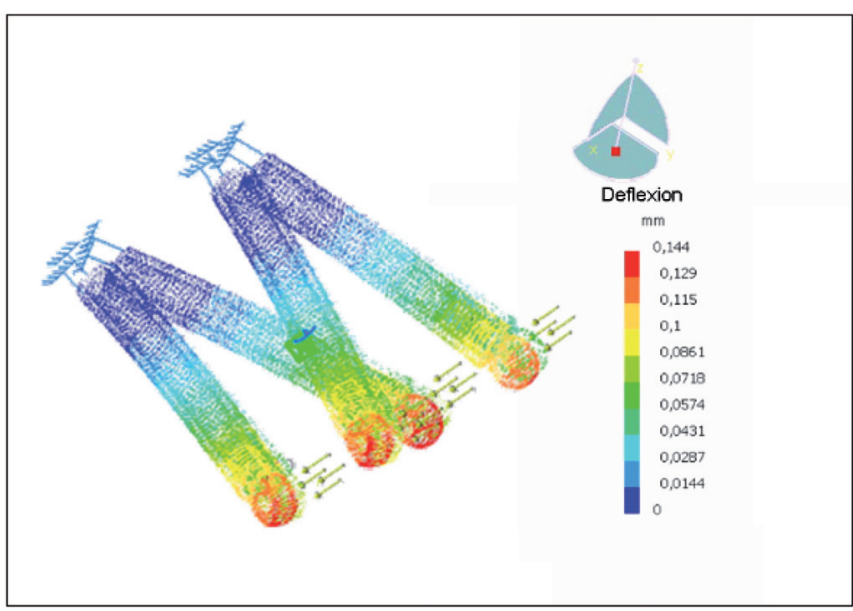

Fig. 7. Deflection representation using CATIA v5.

be lower than $R e / n$ (Eq. (6)). Where $R e$ and $n$ are respectively the elastic limit and the safety margin

$$
\alpha_{\max } \leqslant \frac{R e}{n} .
$$

The first step allows the highest load applied to the lower triangle at point A to be identified (the respective values of the load vectors in $X$ and $Y$ directions: - $1792 \mathrm{~N} ;-1482 \mathrm{~N}$, in a constant deceleration phase, value $\gamma=10 \mathrm{~m} / \mathrm{s}^{2}$ corresponding to an emergency braking condition). However, this calculation identifies the front tie-rod of the lower triangle as the most highly stressed component. During the second step, we determine the normal and tangential load components in the front tie-rod and the corresponding stresses and strains.

As defined previously, during the project, requirements, technical parameters, objective functions, constraints and expert rules are integrated by the expert designers into the ACSP PLM environment and directly associated to the product part list. This data is then extracted and structured in order to create:

- knowledge archives in a knowledge management system, associated to ACSP;

- script files which automatically generate a CATIAv5 parametric CAD model and the Excel Files which describe the product parametric architecture without any solid features. These solid features will then be created by CAD designers, using the previous product architecture;

- the FEM model integrated into the CATIA v5 FEM Software. In this step, the new parameters connected with the design rules and the objective functions are 


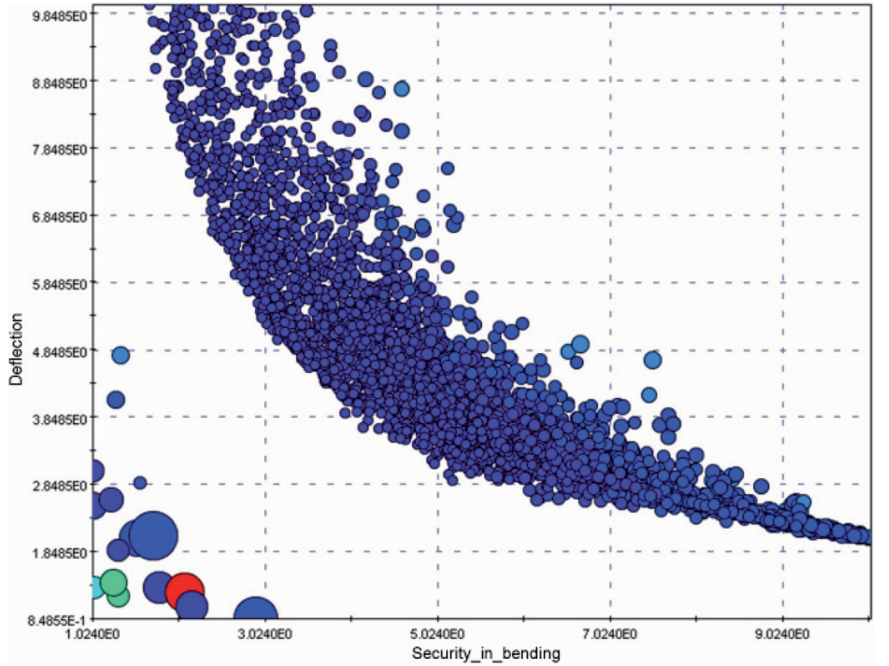

Fig. 8. Admissible solutions in the criteria space: deflection / safety in bending.

checked in the software, for instance, we can check the deflection of the bar. The Figure 8 represents graphically the deflection using the FEM code integrated in CATIA v5.

With this method, we are now able to generate an output MS Excel file with the new calculated FEM constraints at each loop of the optimization algorithm, in order to give an input to the loop and perform the optimization computing process.

In Figures 8 and 9, we obtain the admissible solutions in the Bi-criteria space domain for deflection and safety in bending and then the Pareto curve at the end of the optimization process. Here, we observe the uniform distribution of the Pareto points in the criteria space with 3 functions: deflexion, safety in bending and the weight of the bar.

\section{Conclusions}

To conclude, parametric geometrical models stored in PLM systems and connected to knowledge-based engineering systems, for functional product design and automatic generation of design solutions, are becoming a reality in industry in order to improve productivity and quality in routine design engineering processes. Moreover these automatically generated solutions are not optimized taking into account the various, and sometimes contradictory design objectives and design constraints that must be considered in each project.

In this paper we have experimented with a direct, multi-objective optimization approach in order to help the designer involved in these routine design processes, to choose as quickly as possible the optimal design solutions, considering all the expert design rules, the objective functions (to be minimized or maximized) and also the design constraints.

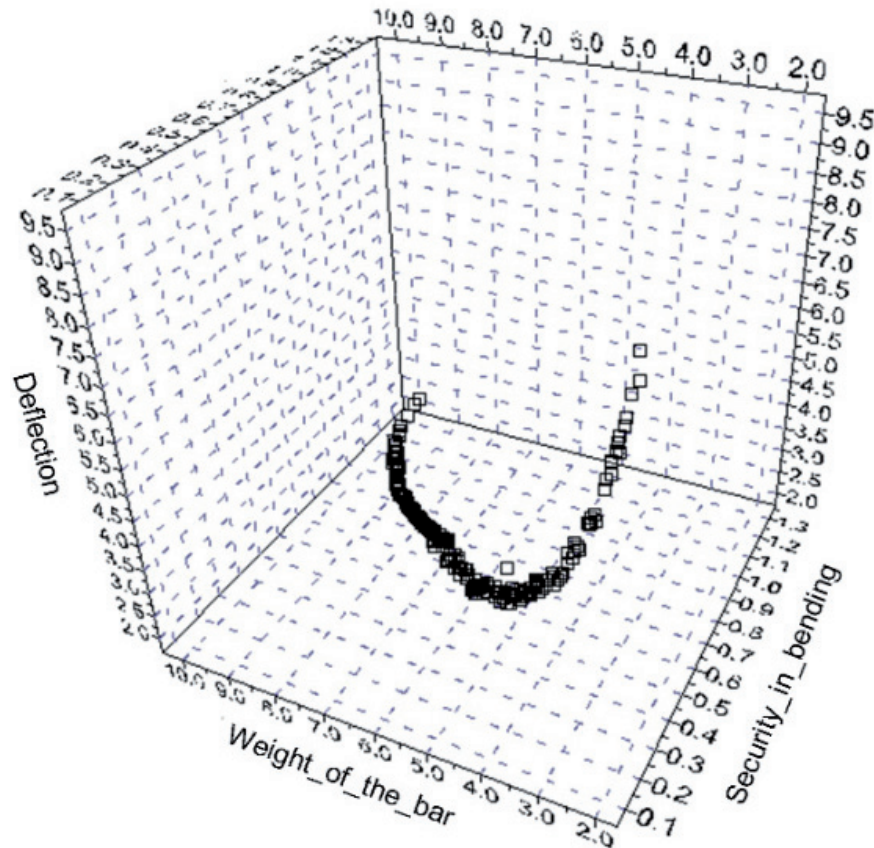

Fig. 9. Pareto domain in 3D with all functions: weight of the bar / safety in bending / Deflection.

We have also highlighted some difficulties related to multi-objective optimization and the handling method used in our genetic algorithm program. This approach has demonstrated its efficiency in the case of our model (the ground-link system of a racing car) in the obtaining of a uniform spray of the Pareto solutions.

The next step would be to improve the software design chain, in order to reduce time devoted to data exchange between the different software types involved in our partially automatic design methodology of optimal solutions (Product Lifecycle Management, Computer Aided Design, Knowledge Based Engineering, Finite Element modelling and simulation and Multi-objective Optimization).

The authors would like to thank Metropolitan Community of the region of Montbéliard, Franche-Comté Region Council, OSEO innovation, French Ministry of industry and the Automotive of the Future cluster, for their funding of this research activity.

\section{References}

1. S. Pugh, Total Design - Integrated Methods for Successful Product Engineering, 1st edn. (Addison-Wesley Publishing, 1990), p. 278

2. G. Pahl, W. Beitz, translated by K. Wallace, L. Blessing, F. Bauert, edited by K. Wallace. Engineering Design, A Systematic Approach. 2nd edn. (Springer-Verlag, New-York, 1995)

3. D. Ullman, The mechanical design process (MacGraw-Hill Higher Education, 2002), p. 409

4. D.E. Whitney, Q. Dong, J. Judson, C. Mascoli, Introducing Knowledge-Based Engineering into an 
Interconnected Product development process. Procedings of the 1999 ASME Design Engineering Technical Conference, September, Las Vegas, Nevada. DET99DTM8741, 1999

5. E. Rechting, Systems Architecting (Pentice Hall, NewYork, NY, USA, 1991)

6. J. Eggers, D. Feillet, S. Kehl, M.O. Wagner, B. Yannou. European Journal of Operational Research 15 (2002)

7. D. Brissaud, O. Garro. Concurrent Engineering: Research and Applications 4, 303 (1996)

8. T. Kvan. Automation in Construction 9, 409 (2000)

9. W. Shen. Computers in Industry 52, 1 (2003)

10. D.T. Liu, X.W. Xu. A Review of Web-based Product Data Management Systems. Computers in Industry 44, $251(2001)$

11. S. Gomes, J.C. Sagot. A concurrent engineering experience based on a cooperative and object oriented design methodology, Integrated Design and Manufacturing in Mechanical Engineering (Kluwer Academic Publishers, Dordrecht, 2002)

12. S. Gomes, J.B. Bluntzer, J.-C. Sagot. Functional design through a PLM system for fastening routine definition of CAD models. PLEDM 2006, Playa Del Carmen, Mexico, 26 November - 1st December, 9p 2006

13. J.H. Holland, Adaptation in natural and artificial systems (University of Michigan Press, Ann Arbor, 1975)

14. D.E. Goldberg, Genetic algorithms in search, optimisation, and machine learning (Addison-Wesley, New York, 1989)

15. Z. Michalewicz, Genetic Algorithms + Data Structures $=$ Evolution (Springer-Verlag, Heidelberg, 1994)
16. K. Deb, D.E. Goldberg, An investigation of niche and species formation in genetic function optimization, edited by J.D. Schaffer, Proceedings of the Third International Conference on Genetic Algorithms (Morgan Kauffman, San Mateo, 1989), pp. 42-50

17. K. Deb, R.B. Agrawal. Complex Systems, 115 (1995)

18. K. Deb, A. Patrap, S. Agarwal, T. Meyarivan. A fast and Elitist multi-objective Genetic algorithm : NSGA-II, Technical Report No. 200001 (Kanpur Genetic Algorithms Laboratory, India Institute of Technology, 2005)

19. F.X. Irisarri, D.H. Bassir, J.F. Maire, N. Carrere. Multiobjective stacking sequence optimisation strategy for laminated composite structures, First international conference on multidisciplinary optimization and applications (EDP Sciences), ISBN 978-2-7598-0023-0, 2007

20. D.H. Bassir, J.L. Zapico, M.P. González. International Journal of Simulation and Multidisciplinary Design Optimization 1, 37 (2007)

21. K. Deb. International Journal of Simulation and Multidisciplinary Design Optimization 1, 1 (2007)

22. M. Domaszewski, D.H. Bassir, W.H. Zhang, Stress displacement and weight minimization by multicriteria optimization and game theory approach, Computer Aided Optimum Design of Structures OPTI 99, Orlando, Florida, USA, WIT Press, 171 (1999)

23. W.H. Zhang, M. Domaszewski, C. Fleury. Int. J. Numer. Meth. Eng. 52, 889 (2001)

24. I. Das, Non linear multicriteria optimization and robust optimality, Ph.D. Thesis, Rice University, Houston, USA, 1997 